\title{
ANTARCTIC OZONE DECREASES: A DYNAMICAL CAUSE?
}

\author{
J. D. Mahlman and S. B. Fels
}

Geophysical Fluid Dynamics Lab/NOAA, Princeton, New Jersey 08542

Abstract. A hypothesis is advanced that natural dynamical processes might explain much of the observed late winter ozone decreases over Antarctica. For this to be the case, sometime after 1979 there must have been a substantial reduction of the wintertime planetary-scale disturbance activity in the Southern Hemisphere troposphere. The expected stratospheric response to such a natural process is to reduce wintertime polar ozone, prolong the life of the polar vortex, reduce the transport of ozone out of the middle stratosphere, and to increase the possibility of polar rising motion shortly after the return of the sun to high latitudes. All of these effects are in qualitative agreement with the observed ozone changes.

\section{Introduction}

The large October decreases of total ozone in Antarctica reported by Farman et al. (1985) have attracted considerable attention. The early attempts to explain the phenomenon have mainly been chemically oriented (Farman et al., 1985; Solomon et al., 1986; McElroy, et al., 1986; Callis and Natarajan, 1986; Tung et al. 1986). To date, these various chemical ozone destruction hypotheses, although ingenious, appear to have important 11mitations.

The only other possible explanations involve dynamical mechanisms. It is reasonable to explore dynamical possibilities simply because most of the high latitude ozone resides in the lower stratosphere, a region where chemical time scales are thought to be much longer than those of transport. We explore here possibilities and data constraints offered by a proposed dynamical explanation of the Antarctic ozone decrease. What we present is necessarily qualitative at this early stage.

The Farman et al. (1985) data for Halley Bay suggest important ozone decreases over at least a 10-year period. However, a larger-scale interpretation is difficult because the ozone minimum inside the winter polar vortex is known to meander from year to year. In this context, note that the satellite data given in Stolarski, et al. (1986) for the polar cap in October suggests nearly constant values through 1979 with a sharp drop thereafter. It is this latter perspective on the ozone decreases that we will invoke here in seeking a dynamical explanation.

Most of the emphasis so far has been directed toward the large October decreases within the polar vortex itself. However, we must also address the ozone changes outside the polar vortex and at other times of the year. For example, Stolarski et a1. (1986) note about a $10 \%$ decrease in October total ozone near the local

This paper is not subject to U.S. copyright. Published in 1986 by the American Geophysical Union.

Paper number $6 \mathrm{~L} 6341$. midlatitude maximum region over the past six years. According to Callis and Natarajan (1986), February data for Halley Bay show declines of order $15 \%$.

Dynamical Requirements and Transport Implications

In this section we address the dynamical mechanisms that must have been at work since 1980 if such processes can contribute to the observed ozone decreases. Presently, the arguments are at best only semi-quantitative, but they are guided by a variety of modeling experiences.

Basic Necessary Requirement: Sometime after 1979 there must have been a substantial reduction of the wintertime planetary-scale disturbance activity in the Southern Hemisphere troposphere.

This stated requirement is rather imprecise. More specifically, what we have in mind here is a reduction in the disturbance-induced zonal body force on the westerly flow of the winter lower stratosphere as measured by the Eliassen-Palm (EP) flux convergence. A recent analysis is consistent with this, showing a marked reduction since 1979 of the September-mean vertical component of the EP flux at $100 \mathrm{mb}$ from $20^{\circ} \mathrm{S}-90^{\circ} \mathrm{S}$ (Nagatani, R. M., and A. J. Miller, "The influence of lower stratospheric forcing on the October Antarctic ozone decrease", in preparation). Given this evidence that the Basic Necessary Requirement may have been satisfied, we discuss below some expected stratospheric consequences. They are:

a. An increase in the length of Antarctic "winter", as measured by increased duration of a colder polar vortex.

b. A general (non-seasonal) reduction of the polar ozone column and a flattenting of the meridional slopes of tracer isolines relative to Isentropic surfaces.

c. A decrease in the hemispheric mean ozone.

d. A transient decrease in the polar ozone column as solar heating returns in late winter. We now discuss the reasons behind these expected changes. Given the Basic Necessary Requirement, we would first expect the decreased magnitude of EP flux convergence to lead to a stronger polar night westerly wind. This decrease should lead to a reduction of the (eddy forced) residual (approximately diabatic) circulation (Andrews, et al., 1983, App. B). Consistent with the strengthened zonal winds and decreased (downward) residual circulation is a colder polar vortex (Consequence " $a$ "). Associated with such a cold polar vortex should be increased polar stratospheric clouds (PSC's). This assumed decrease of tropospheric forcing should lead to decreased meridional slopes of quasi-conserved trace gases such as chlorofluorocarbons, $\mathrm{N}_{2} \mathrm{O}$ or ozone, when the slopes are measured relative to isentropic surfaces 


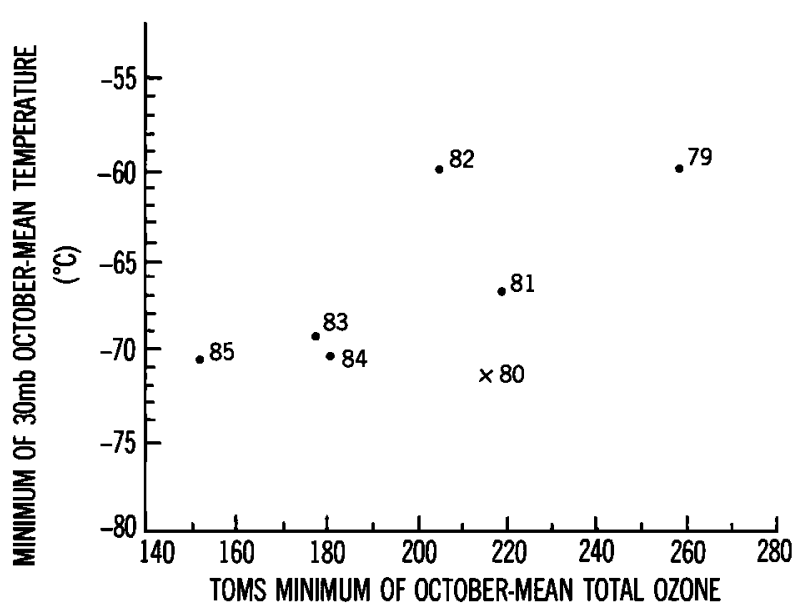

(DOBSON UNITS)

F1g. 1. P1ot of October-mean minimum of $30 \mathrm{mb}$ temperature (ordinate) and total ozone (abscissa). Temperature is taken from the National Meteorological Center (NMC) analysis (courtesy of A. J. Miller); ozone is from the TOMS satellite (courtesy of R. S. Stolarski). Note the apparent relation between lower total ozone and lower polar vortex temperatures. The 1980 point is plotted with an " $x$ " because of uncertainties in the MMC analysis at that time.

(Consequence "b"). According to Mahlman et al. (1986), the equilibrium meridional slope of a nearly conserved trace constituent is roughly equal to the mean high-latitude downward diabatic advection velocity divided by the effective meridional diffusion velocity. Both of these processes are caused by tropospheric disturbances propagating into the stratosphere and dissipating. However, modeling experience indicates that as the effective tropospheric forcing decreases, the diabatic circulation reduces by a greater factor than does the effective meridional diffusion velocity. Thus, the meridional slopes of trace constituent isolines should become flatter relative to isentropic surfaces. Such flatter slopes imply that stratospheric source gases such as ozone, odd chlorine or odd nitrogen should be lower near the poles in the lower stratosphere.

In this context, note that the previous $3-D$ modeling study of Mahlman et al. (1980) simulated very low late winter values of Antarctic ozone, rather near the values now observed. In that paper the low values were Identified as a serious model defect. We now know that the general circulation model (GCM) providing the winds for that ozone calculation was deficient in its dynamical forcing of the stratosphere (Manabe and Mah1man, 1976). The $\mathrm{N}_{2} \mathrm{O}$ study of Mahlman et al. (1986), using the same parent GCM, also shows $\mathrm{N}_{2} \mathrm{O}$ meridional slopes that are somewhat too flat relative to observations. That model and observations also indicate that the Southern Hemisphere $\mathrm{N}_{2} \mathrm{O}$ meridional slopes are flatter than those of the Northern Hemisphere. Thus, the climatological polar Southern Hemisphere winter is regarded to be less "dynamical" than that of the Northern Hemisphere. By inference, the recent (1980-85) polar Southern Hemisphere winter must have been even less so.

Any valid theory must explain why the ozone decreases are more pronounced in September and 0ctober. In this context, note that the observed decreases may be regarded as partially a "ledge" phenomenon. That is, large ozone increases are observed in association with the final breakup of the polar vortex. Thus, any dynamical process that contributes to the extension of "winter" (1.e., the integrity of the polar vortex) can contribute to a secular decrease in late winter ozone simply by stalling the time of onset of the larger spring increases (Consequence " $a$ ").

That is effectively what should be expected in a "less dynamical" Antarctic polar winter. The lessened amplitudes of tropospheric disturbances would lead to a reduced late winter "erosion" of the polar vortex (McIntyre and Palmer, 1983). Thus, the final end of "winter" will be extended closer to the point where solar radiation directly destroys the cold pool, irrespective of disturbance-induced irreversible mixing.

A check on these expectations can be obtained by comparing the evolution of ozone and temperature in the polar vortex. Figure 1 shows TOMS total ozone of the minimum monthly mean value for each October from 1979-85 plotted against the minimum value of October monthly mean $30 \mathrm{mb}$ temperature. Figure 1 indicates that the deep ozone minimums of the later years are assoctated with colder temperatures. The correlation between the temperature and ozone data in Figure 1 is +0.59 . If the questionable 1980 point is removed, this increases to +0.78 (significant at 95\% confidence). The dynamical mechanism may thus be playing a key role in producing observed ozone drops. Figure 1 suggests roughly a 10 Dobson unit drop in total ozone for each $1^{\circ} \mathrm{C}$ decrease in October polar vortex temperature. If this interpretation is correct, the Antarctic late winter ozone could make a spectacular comeback should the tropospheric forcing return to its assumed earlier levels.

Another feature that the dynamical hypothesis must explain is the apparent systematic downward trend since about 1979. Suppose that the dynamical change was essentially discontinuous starting in about 1980. If high latitude, lower stratospheric ozone is determined mainly by transport out of the middle stratosphere, the adjustment to lesser winter ozone values could take substantially longer than one year. The lower stratosphere has to "clear out" the excess ozone that is no longer transport-compatible with the reduced forcing. In the $3-D$ ozone experiment of Mahlman et al. (1980), the Initial condition had considerably more total ozone than did the eventual equilibrium solution. Between year 2 and year 3 in that model, the October mean total ozone at $84^{\circ} \mathrm{S}$ dropped $18 \%$; between year 3 and year 4 it dropped $12 \%$ more (at the equator it dropped on $1 y$ 5\%). Thus, high latitude total ozone is likely to decrease over several years due to a sharp decrease in tropospheric forcing.

A second possibility is that the tropospheric forcing itself has diminished systematically over a few years interval (see Nagatani and Miller, (1986). It is interesting to note that the lowest October $30 \mathrm{mb}$ polar vortex temperatures were recorded in 1983, 1984 and 1985. The above two processes combined could act to produce an apparent downward trend over a number of years. These arguments also suggest that Antarctic ozone could return sharply in the near future.

Another expectation is that a systematic ozone decrease over the entire hemisphere should have 
occurred in response to diminished tropospheric forcing. The mechanistic models of Mahlman et a1. (1986) and Holton (1986) predict that the hemispheric average vertical transport efficiency (1-D diffusion coefficient) is proportional to the square of the intensity of the diabatic meridional circulation. Thus, we might expect that, as the transport efficiency diminishes, there will be a lessened net supply of ozone to the lower stratosphere (Consequence "c").

\section{The September Ozone Decrease: Return of the}

\section{Flywheel?}

An important problem that remains unaddressed is the indication of a significant ozone loss in the polar vortex in early September. This transient loss is in addition to any secular decrease in ozone due to the prolongation of winter discussed above.

It thus is important to establish how much of the recent decrease is associated with early September insolation, and how much is already present in the polar night. Useful data from the Nimbus 7 SBUV instrument for 14-day averaging intervals at $80^{\circ} \mathrm{S}$ has been compiled by $\mathrm{Dr}$. R. Stolarski. The data indicate that, for the 5-year mean from 1979-1983, the minimum occurs during the 1nterval 15-28 September. Also, the largest decreases between 1-14 and 15-28 September occur in the later years. Thus, an important fraction of the ozone decrease comes after the return of the sun. During 1982 and 1983 , the total column decreased by about $10 \%$ between the 1-14 and 15-28 September averaging intervals. Because the ozone mixing ratio increases upward, the usual eddy-driven polar descending vertical motion would act to produce total column increases. Moreover, because the "ozone hole" is a minimum in the horizontal, irreversible horizontal eddy exchange processes also tend to produce polar ozone increases. In fact, it is just these exchange processes that produce the pronounced polar ozone increases in November associated with the final breakup of the polar vortex.

We now seek a mechanism for producing rising motion at the pole during early September that may have become more important in recent years. Data from the Syowa station suggest a mean vertical ozone gradient of $0.23 \mathrm{ppmv} / \mathrm{km}$ near $15 \mathrm{~km}$, where the mixing ratio is about 1 ppmv. The upward velocity required to produce a $10 \%$ column decrease over two weeks is thus about $30 \mathrm{~m} /$ day. This small vertical velocity corresponds to an adiabatic cooling rate of about $0.3 \%$ day. Since solar ozone heating at $15 \mathrm{~km}$ increases from $0.1 \%$ day to about $0.3 \%$ day during September at $80^{\circ} \mathrm{S}$, it is natural to ask whether strongly varying net diabatic heating might not give rise to the required vertical motion. This has already been suggested by Tung et al. (1986), based on the work of R. Snieder and S. B. Fels ("The flywheel effect in the middle atmosphere." In preparation).

The flywheel model determines how transient diabatic heating acting on an eddy-free, geostrophically balanced zonal flow is partitioned between temperature rise and adiabatic cooling. In such a radiatively determined regime, any change in meridional temperature gradient must result in a change in the zonal wind; this will be resisted by the mechanical inertia of the air. Thus, strong meridional gradients in solar heating can result in vertical motion, as well as temperature changes. This mechanism thus might provide the required vertical velocity ( $w$ ) (Consequence " $d$ ").

Unfortunately, the required result is not obtained easily. The induced vertical velocity w is a non-local function of the transient net radiative heating. An estimate of its magnitude can be obtained by considering the artificial situation in which the solar heating ( $\mathrm{J}_{\text {solar }}$ ) varies harmonically in time with angular frequency $\omega$ and longwave cooling is characterized by a relaxation time $\tau_{r}$. In this case one finds

$$
|w| \sim J_{\text {solar }} \frac{\mathrm{R}}{\mathrm{HN}^{2}}\left|\frac{1}{1+\left(1-\frac{1}{1 \omega \tau}\right) \frac{\mathrm{L}^{2} \mathrm{f}^{2}}{\mathrm{D}^{2} \mathrm{~N}^{2}}}\right| \text {. }
$$

Here $f$ is the Coriolis parameter, $N$ the BruntVäisälä frequency, $R$ the gas constant, $H$ the scale helght, and $L$ and $D$ are the horizontal and vertical scales of the heating (Snieder and Fels, 1987).

Equation (1) shows that significant vertical motion is produced by rapidly varying insolation of large vertical and small horizontal scale. Moreover, the model shows that upward motion will occur where the heating is large. In early September, when the pole is still dark, w due to ozone heating should actually be negative there, and only becomes positive after the equinox.

In fact, detailed numerical calculations (Snieder and Fels, 1987) show that at $80^{\circ} \mathrm{S}$, the vertical velocities near $15 \mathrm{~km}$ in the first half of September are 1ndeed negative and small, on the order of -5 to $-10 \mathrm{~m} /$ day. By mid-0ctober, positive velocities of the same magnitude have set in, but these are both too small and too late to explain the September ozone drop. At $69^{\circ} \mathrm{S}$ (the latitude of Syowa), the situation is equally bad because the vertical velocities are less than 5 $\mathrm{m} /$ day during September.

Although these calculations show that ozone heating alone cannot produce the required rising motion, it is possible, however, that PSC's might provide a sultable configuration of heating, as shown schematically in Figure 2. In this case, the solar heating has a sharp maximum near the terminator, resulting in strong upward motion there. A crude estimate based on Equation (1) suggests that a $\mathrm{J}_{\mathrm{solar}}$ of $1 \%$ day, a cloud depth of $10 \mathrm{~km}$, and a horizontal scale of $1000 \mathrm{~km}$ produces vertical velocities of about $50 \mathrm{~m} /$ day.

Should this indeed be the process describing the observed September dip in total ozone, it may have been suppressed prior to recent years. In an epoch dominated by strong winter eddy transport of heat and ozone Into a weaker vortex, the zonally symmetric transition out of winter described above would never have had a chance to take place. In the earlier regime, the cold vortex (and its PSC's) would have broken down sooner due to erosion by mid-latitude eddies. It is only the weakening of the eddies that allows the colder vortex to "live to see the sunrise", and thus enables the flywheel effect to operate.

\section{Summary}

We advance a dynamical hypothesis as a possible explanation for the large late winter 


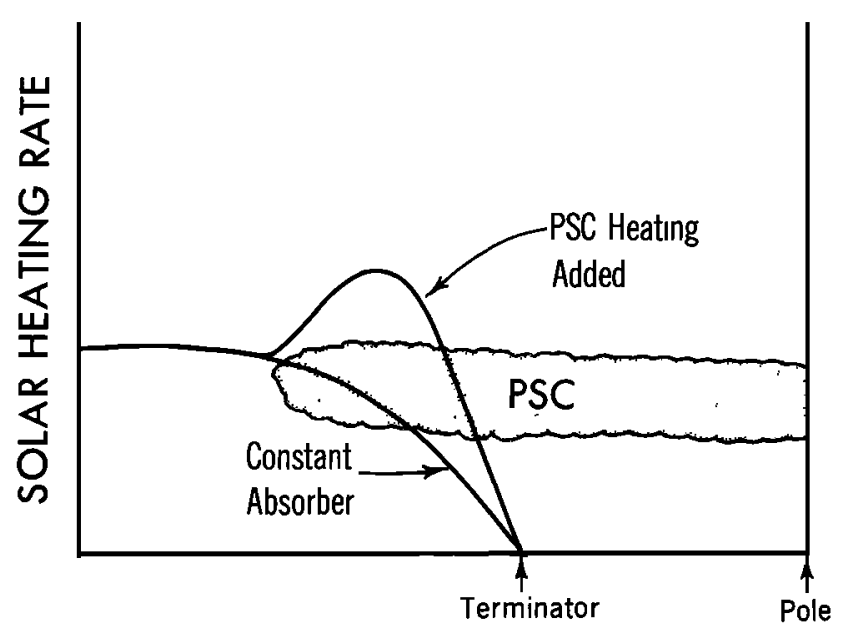

LATITUDE

Fig. 2. Schemat1c illustration of possible 1mpact of polar stratospheric clouds (PSC's) on transient late winter solar heating rates. For a constant absorber, the heating rate peaks at relatively low latitudes. With PSC's present in the cold polar vortex, a maximum heating rate near the terminator is possible.

decreases in Antarctic ozone since about 1980. The hypothesis assumes there has been a significant decrease in the magnitude of Southern Hemisphere winter tropospheric forcing due to planetary-scale disturbances. Observed decreasing "trends" in October polar vortex temperature and September vertical component of EP flux are compatible with this hypothesis.

A number of ozone transport responses to this decreased forcing are expected. The meridional slopes of ozone mixing isolines should be flatter, relative to isentropic surfaces, with lower values at the Pole. The decreased forcing should lead to a longer winter, as manifested by the polar vortex lasting longer.

The systematic downward trend in ozone is expected in part to be due to the several years required for the polar lower stratosphere to come into equilibrium with the new reduced troposphere forcing. Reduced hemispheric-mean ozone amounts are expected because the efficlency of removal of ozone from the middle stratosphere is roughly proportional to the square of the eddy-forced diabatic meridional circulation.

The nearly $10 \%$ drop in total ozone over a 2-week period in September may be due to a radiative-dynamical induced transient rising motion in response to the so-called "flywheel" effect. This mechanism is unable to explain the observed decrease without addition of an additional polar cap absorber. We speculate here that the PSC's (assumed to last longer in this colder, weaker dynamics regime) may provide the required additional absorption.

Finally, none of these arguments presented here preclude significant chemical effects. They do, however, strongly indicate that dynamical factors have been important in shaping the observed character of the phenomenon.

Acknowledgments. We are grateful to K. P. Hamliton, C. B. Leovy, T. N. Palmer, $V$. Ramaswamy and R. Snieder for their scientific guidance. Special thanks are due to $M$. McFarland, A. J. Mtller, S. Solomon, and R. S. Stolarski for their help in providing key observational information.

\section{References}

Andrews, D.G., J.D. Mahlman, and R.W. Sincla1r, Eliassen-Palm diagnostics of wave-mean flow interaction in the GFDL "SKYHI" general circulation model, J. Atmos. Sci., 40, 2768-2784, 1983.

Callis, L. B., and M. Natarajan, The Antarctic ozone minimum: relationship to odd nitrogen, odd chlorine, the final warming and the 11-year cycle, J. Geophys. Res., 91, D10, 10771-10796, $19 \overline{86}$.

Farman, J.C., B.G. Gardiner, and J.D. Shank1in, Large losses of total ozone in Antarctica reveal seasonal $\mathrm{ClO}_{\mathbf{x}} / \mathrm{NO}_{\mathbf{x}}$ interaction, Nature, $315,207-210,1985$.

Holton, J.R., A dynamically based transport parameterization for one-dimensional photochemical models of the stratosphere, J. Geophys. Res., 91, D2, 2681-2685, 1986b.

Mahlman, J.D., H. Levy II, and W.J. Moxim, Three-dimensional tracer structure and behavior as simulated in two ozone precursor experiments, J. Atmos. Sci., 37, 655-685, 1980.

Mahlman, J.D., H. Levy II, and W.J. Moxim, Three-dimensional simulations of stratospheric $\mathrm{N}_{2} \mathrm{O}$ : predictions for other trace constituents, J. Geophys - Res., 91, D2, 2687-2707, 1986.

Manabe, S., and J.D. Mahlman, Simulation of seasonal and interhemispheric variations in the stratospheric circulation, J. Atmos. Sci., 33, 2185-2217, 1976.

McElroy, M.B., R.S. Salawitch, S.C. Wofsy, and J.A. Logan, Reductions of Antarctic ozone due to synergistic interactions of chlorine and bromine, Nature, 321, 759-762, 1986.

Solomon, S., R.R. Garcia, F.S. Rowland, and D.J. Wuebbles, On the depletion of Antarctic ozone, Nature, 321, 755-758, 1986.

Stolarski, R.S., A.J. Krueger, M.R. Schoeberl, R.D. McPeters, P.A. Newman, and J.C. Alpert, Nimbus 7 SBUV/TOMS measurements of the springtime Antarctic ozone hole, Nature (in press), 1986.

Tung, K.K., M.K.W. Ko, J.M. Rodriquez, and N.D. Sze, Are Antarctic ozone variations a manifestation of dynamics or chemistry? Nature, 322, 811-814, 1986.

(Recelved August 20, 1986; accepted September 11, 1986.) 